October 2, 2012

\title{
Classical Yang-Mills theory in condensed matter physics
}

\author{
Bertrand Berche ${ }^{1,2}$ and Ernesto Medina ${ }^{2,1}$ \\ ${ }^{1}$ Statistical Physics Group, P2M, Institut Jean Lamour, UMR CNRS 7198, \\ Nancy Université, BP70239, F-54506 Vandouvre les Nancy, France \\ ${ }^{2}$ Laboratorio de Física Estadística de Medios Desordenados, Centro de Física, \\ Instituto Venezolano de Investigaciones Científicas, Apartado 21874, Caracas 1020-A, Venezuela
}

\begin{abstract}
Recently, gauge field theory approaches were extensively used in order to discuss the physical consequences of spin-orbit interactions in condensed matter physics. An $S U(2) \times U(1)$ gauge theory is very naturally borne out and provides an illustrative example of a classical Yang-Mills field theory at work. This approach may serve as an exemplification of non-Abelian field theories for students in general physics curriculum. It allows to introduce discussions on fundamental ideas like Noether currents, gauge symmetry principle, gauge symmetry breaking and non linear Yang Mills equations in very concrete physical situations that makes it accessible to a broad audience.
\end{abstract}

Résumé. Les théories de jauge ont récemment été utilisées de manière systématique pour discuter les conséquences physiques de l'interaction spin-orbite en physique de la matière condensée. Une théorie $\mathrm{SU}(2) \times \mathrm{U}(1)$ apparaît naturellement et fournit un exemple d'illustration du fonctionnement des théories classiques de Yang et Mills. Cette approche peut servir d'example de théorie de jauge non abélienne pour des étudiants dans un cours de physique générale. Cela permet d'introduire et de discuter les idées fondamentales comme les courants conservés de Noether, le principe de symétrie de jauge, la brisure de la symétrie de jauge et les équations non linéaires de Yang et Mills dans des situations physiques concrètes, les rendant ainsi accessible à une vaste communauté.

\begin{tabular}{|c|c|}
\hline$\varphi(\vec{r}, t)$ & wave function or complex scalar field \\
\hline$\psi(\vec{r}, t)$ & Pauli 2-component spinor or Pauli spinor field \\
\hline$\Psi(\vec{r}, t)$ & Dirac 4-component spinor \\
\hline $\mathscr{L}$ & Lagrangian density \\
\hline$H$ & Schrödinger Hamiltonian \\
\hline $\mathrm{H}$ & Pauli Hamiltonian \\
\hline $\mathscr{D}_{t}, \overrightarrow{\mathscr{D}}$ & $\mathrm{U}(1)$ covariant derivatives \\
\hline$\phi, \vec{A}$ & $\mathrm{U}(1)$ scalar and vector gauge fields \\
\hline$\vec{E}, \vec{B}$ & electric and magnetic fields \\
\hline$\rho(\vec{r}, t)$ & charge density \\
\hline$\vec{j}(\vec{r}, t)$ or $\vec{J}(\vec{r}, t)$ & charge current density \\
\hline$e, g$ & electric and isospin charge \\
\hline$\tau^{a}$ & $\mathrm{SU}(2)$ generators \\
\hline$\alpha$ & $\mathrm{SU}(2)$ contraction $\tau^{a} \alpha^{a}$ \\
\hline $\mathscr{D}_{t}, \mathscr{D}$ & SU(2) covariant derivatives \\
\hline $\mathbf{s}^{a}$ & $a$-component of the spin $\frac{1}{2}$ operator \\
\hline$\sigma^{a}$ & $a-$ component Pauli matrix \\
\hline$\chi=\frac{1}{2} \sigma^{a} \chi^{a}, \overrightarrow{\mathbf{W}}=\frac{1}{2} \boldsymbol{\sigma}^{a} \overrightarrow{\mathrm{W}}^{a}$ & SU(2) scalar and vector gauge fields \\
\hline $\overrightarrow{\mathbf{E}}=\frac{1}{2} \sigma^{a} \overrightarrow{\mathrm{E}}^{a}, \overrightarrow{\mathbf{B}}=\frac{1}{2} \boldsymbol{\sigma}^{a} \overrightarrow{\mathrm{B}}^{a}$ & $\mathrm{SU}(2)$ field strengths \\
\hline $\begin{array}{l}s_{\text {mat. }}^{a}(\vec{r}, t), \rho_{\text {mat. }}^{a}(\vec{r}, t), \vec{J}^{a}(\vec{r}, t) \\
s_{\text {rad }}^{a}(\vec{r}, t), \rho_{\text {rad. }}^{a}(\vec{r}, t), \vec{J}^{a}(\vec{r}, t) \\
\vec{s}(\vec{r}, t)\end{array}$ & $\begin{array}{l}a \text {-component of the matter spin or isospin density and current density } \\
a \text {-component of the radiation spin or isospin density and current density } \\
\text { vector spin density }\end{array}$ \\
\hline
\end{tabular}

\section{INTRODUCTION}

Yang-Mills theories are usually introduced to students in the context of quantum field theory, but are never encountered in their classical form at the undergraduate level [1]. Regarding the fundamental role of gauge symmetry in the construction of physical theories, this is desirable in order to make the underlying concepts accessible to physics students early in the curriculum. In the abstract of a paper by R. Mills [2], the content of gauge theories is summarized as follows: "...the gradual emergence of symmetry as a driving force in the shaping of physical theory; the elevation of Noether's theorem, relating symmetries to conservation laws, to a fundamental principle of nature; and the force idea ("the gauge principle") that the symmetries of nature, like the interactions themselves, should be local in character." This obviously brings the concept of gauge 
invariance to the level of a keystone principle among physical theories, rather than a specific property of electromagnetic theory.

The non relativistic limit of Dirac equation, namely the Pauli equation and its corrections to Schrödinger theory, i.e. the spin-orbit (SO) interaction and the Zeeman interaction, offers a natural frame for an SU(2) gauge field theory. This does not only give an elegant formulation for the coupling of spin degrees of freedom with external electromagnetic fields, but also the Yang-Mills approach provides natural answers to fundamental issues such as conservation laws which may offer illuminating insights in the condensed matter physics context [3]. Here we show that it may be a useful and very concrete way to introduce Yang-Mills theory, in the condensed matter physics curriculum.

A reformulation of the spin-orbit coupling Hamiltonian in terms of non-Abelian gauge fields [4-6] was explicitly given in ref. [7-13] where the SO interaction is presented as a $\mathrm{SU}(2) \times \mathrm{U}(1)$ gauge theory. A key property for that purpose is to deal with an SO interaction which is linear in the momentum, $H_{S O} \sim \vec{p} \cdot(\vec{\sigma} \times \vec{E})$ in such a way that it can be absorbed in the kinetic energy through a minimal coupling procedure, $\vec{p} \longrightarrow \vec{p}-\vec{\sigma} \times \vec{E}$, up to unessential numerical factors. This property is fulfilled by the Pauli Hamiltonian, but also by the Rashba SO interaction [14] and by the two-dimensional reduction of the Dresselhaus SO interaction [15]. A potential realization of such situation in the general case is thus the 2-dimensional electron gas (2DEG) as encountered in semiconductor hetero-junctions [16] and 2DEG quantum rings [17].

Such gauge point of view, in more general terms, has been known for some time[18-20]. This formulation is very revealing, since the consistent gauge structure of the theory becomes obvious and the physics of spin currents, persistent currents and color diamagnetism[21] can be understood in a manner analogous to the well known U(1) gauge theories.

The plan of the paper is as follows: we start in section $\amalg$ with the case of electromagnetic theory which will then serve as a basis to the non-Abelian Yang-Mills theory presented in section III Section IV discusses, in this context, the case of spin-orbit and Zeeman interactions and application to Einstein-de Haas effect is discussed in section $\mathbf{V}$ We have decided to avoid most of the time the use of tensor analysis and instead embrace vector algebra, in order to obtain equations of motion in a form very similar to Maxwell equations in matter, as they are known in standard textbooks [22]. We feel that this will help the part of the community who is less familiar with field theory, appealing to the broader intuition from electromagnetism.

\section{A WELL-KNOWN GAUGE FIELD THEORY: THE EXAMPLE OF ELECTROMAGNETISM}

In this section, we collect the standard results of classical gauge field theory applied to electromagnetism in order to later follow a parallel approach in the case of non Abelian and SO interaction.

\section{A. Conserved currents}

A fundamental question in physics poses the problem of the electric charge. Why is charge conserved? Historically we have made the empirical observation that in all fundamental processes, e.g. collisions between elementary particles, the electric charge is conserved. The sum of the charges of the outgoing particles is always equal to the sum of the charges of the incoming particles. This property then translates into a hydrodynamic conservation equation $\partial_{t} \rho(\vec{r}, t)+\vec{\nabla} \cdot \vec{j}(\vec{r}, t)=0$, where $\rho(\vec{r}, t)$ is the electric charge density calculated on a coarsed grained scale when a continuous density make sense. Integration over a mesoscopic (or macroscopic) scale, then leads to $\frac{d Q(t)}{d t}=\int d^{3} r \partial_{t} \rho(\vec{r}, t)=-\oint \vec{j}(\vec{r}, t) \cdot d \vec{S}=0$ provided that the volume of integration is large enough and the current densities vanish at the boundaries. So conservation of charge follows from an experimental observation, but its origin is not something obvious. An answer to the question of the origin of such a conservation law (formulated here in a provocative manner) is that Hilbert spaces and the complex fields living in these spaces don't associate physical relevance to global phases of such fields. Here we refer to the fact that in Quantum Mechanics (QM) the results of measurements are given by the matrix elements $\langle\varphi|O b s.| \varphi\rangle$ of Hermitian operators Obs. associated to physical observables, and such matrix elements are independent of the choice of reference for the phase of the complex vector $\varphi$.

The principle of conservation of the electric charge, experimentally legitimate, is derived using the second approach from a more fundamental and more abstract principle of invariance of the theory with respect to global phase changes. The main advantage of the second answer is that it enables us to connect to other similar theories. More importantly, it leads to a consistent construction of the electromagnetic theory with no a priori knowledge other than that implied by the Lagrangian formalism which is the language adapted for such approaches. This is what we now show. 
The Schrödinger equation follows from a least action principle [23]

$$
\frac{\delta}{\delta \varphi^{*}} \int \mathscr{L}_{0} d^{3} r d t=\frac{\partial \mathscr{L}_{0}}{\partial \varphi^{*}}-\partial_{k} \frac{\partial \mathscr{L}_{0}}{\partial\left(\partial_{k} \varphi^{*}\right)}-\partial_{t} \frac{\partial \mathscr{L}_{0}}{\partial\left(\partial_{t} \varphi^{*}\right)}=0
$$

where the free matter Lagrangian density $\mathscr{L}_{0}$ is an ordinary function $\mathscr{F}$ of the wave function $\varphi(\vec{r}, t$ ) (a complex scalar field describing matter), its space and time derivatives, and the corresponding complex conjugates treated as independent variables. $\mathscr{L}_{0}=\mathscr{F}\left(\varphi, \varphi^{*}, \vec{\nabla} \varphi, \vec{\nabla} \varphi^{*}, \partial_{t} \varphi, \partial_{t} \varphi^{*}\right)$ is the Jordan-Wigner Lagrangian density [23, 24],

$$
\mathscr{L}_{0}=i \hbar \varphi^{*} \partial_{t} \varphi-\frac{\hbar^{2}}{2 m}(\vec{\nabla} \varphi)^{*}(\vec{\nabla} \varphi)-V \varphi^{*} \varphi
$$

and equation (1) applied to this Lagrangian recovers the Schrödinger equation. As discussed above, Quantum Mechanics is invariant under a global change of phase of the wave function

$$
\varphi(\vec{r}, t) \rightarrow \exp (\text { ie } \alpha / \hbar) \varphi(\vec{r}, t), \quad \alpha=\text { const. }
$$

since all physical quantities, expressed in terms of matrix elements, are independent of the global phase choice $\alpha$. The coefficient $e / \hbar$ in the phase is present for later convenience. It simply fixes the dimensions of the $e \alpha$ to those of an action. The phase transformation above is a continuous symmetry ( $\alpha$ can take any real value) which leaves the Lagrangian density unchanged. Applying Noether theorem to an infinitesimal gauge transformation $\varphi \rightarrow \varphi^{\prime} \simeq(1+i e \alpha / \hbar) \varphi=\varphi+\delta \varphi[23$, 25], it follows that we can define a conserved current obeying a continuity equation. Indeed, demanding the global gauge transformation to be a symmetry, i.e. to preserve the lagrangian density $\mathscr{L}_{0}$ unchanged leads to

$$
\begin{aligned}
\delta \mathscr{L}_{0} & =0=\frac{\partial \mathscr{L}_{0}}{\partial \varphi} \delta \varphi+\frac{\partial \mathscr{L}_{0}}{\partial \dot{\varphi}} \delta \dot{\varphi}+\frac{\partial \mathscr{L}_{0}}{\partial \vec{\nabla} \varphi} \delta \vec{\nabla} \varphi+\left(\varphi \rightarrow \varphi^{*}\right) \\
& =-\frac{i e \alpha}{\hbar}\left[\partial_{t}\left(\varphi^{*} \frac{\partial \mathscr{L}_{0}}{\partial \dot{\varphi}^{*}}-\frac{\partial \mathscr{L}_{0}}{\partial \dot{\varphi}} \varphi\right)+\vec{\nabla} \cdot\left(\varphi^{*} \frac{\partial \mathscr{L}_{0}}{\partial \vec{\nabla} \varphi^{*}}-\frac{\partial \mathscr{L}_{0}}{\partial \vec{\nabla} \varphi} \varphi\right)\right] \\
& =-\alpha\left(\partial_{t} \rho+\vec{\nabla} \cdot \vec{j}\right)
\end{aligned}
$$

where use has been made of the equations of motion (1) in the second line. This leads to the definition of a charge density $\rho(\vec{r}, t)=e \varphi^{*}(\vec{r}, t) \varphi(\vec{r}, t)$ and a charge current density $\vec{j}(\vec{r}, t)=\frac{-i e \hbar}{2 m}\left(\varphi^{*}(\vec{r}, t) \vec{\nabla} \varphi(\vec{r}, t)-\varphi(\vec{r}, t) \vec{\nabla} \varphi^{*}(\vec{r}, t)\right)$. This very interesting connection between "phase invariance" in QM and charge conservation was first made by F. London. For interested readers, historical aspects of gauge invariance are discussed at length in the book of O'Raifeartaigh [26] or in the reviews [27, 28].

\section{B. The gauge invariance principle illustrated}

Let us now quote Salam and Ward [29], (see also Novaes [30]), who speculated on whether a local generalization of the gauge invariance would generate strong, weak and electromagnetic interactions and the associated new fundamental conservation laws: "Our basic postulate is that it should be possible to generate strong, weak, and electromagnetic interaction terms ... by making local gauge transformations on the kinetic-energy terms in the free Lagrangian for all particles." The message behind this sentence is that theories which have a larger symmetry class (local gauge symmetry) have richer dynamical structure (new interactions present), and the power of gauge field theory appears when one assumes that the phase invariance invoked above also holds in the case of local phase changes $\alpha(\vec{r}, t)$,

$$
\varphi(\vec{r}, t) \rightarrow \exp (i e \alpha(\vec{r}, t) / \hbar) \varphi(\vec{r}, t) \equiv G \varphi(\vec{r}, t)
$$

where $G \equiv G(\vec{r}, t)$. This is obviously not a symmetry of the original Lagrangian density $\mathscr{L}_{0}$, since $\vec{\nabla} \varphi(\vec{r}, t)$ and $\partial_{t} \varphi(\vec{r}, t)$ do not transform like the wave function itself, and as a consequence, terms like $i \hbar \varphi^{*} \partial_{t} \varphi$ or $-\frac{\hbar^{2}}{2 m} \vec{\nabla} \varphi^{*} \vec{\nabla} \varphi$ are not gauge invariant (the first of these terms for example transforms into $\left.i \hbar \varphi^{*} \partial_{t} \varphi-e\left(\partial_{t} \alpha\right) \varphi^{*} \varphi\right)$. In order to repair this lack of symmetry of the original Lagrangian, and to be able to build a new one with the expected invariance property, one may define so-called covariant derivatives $\overrightarrow{\mathscr{D}}=\vec{\nabla}-\frac{i e}{\hbar} \vec{A}(\vec{r}, t)$ and $\mathscr{D}_{t}=\partial_{t}+\frac{i e}{\hbar} \phi(\vec{r}, t)$ in such a way that acting on $\varphi(\vec{r}, t)$, they transform like the 
wave function, i.e. according to $\overrightarrow{\mathscr{D}} \varphi(\vec{r}, t) \rightarrow \exp (i e \alpha(\vec{r}, t) / \hbar) \overrightarrow{\mathscr{D}} \varphi(\vec{r}, t)$ and $\mathscr{D}_{t} \varphi(\vec{r}, t) \rightarrow \exp (i e \alpha(\vec{r}, t) / \hbar) \mathscr{D}_{t} \varphi(\vec{r}, t)$. The two fields $\vec{A}(\vec{r}, t)$ and $\phi(\vec{r}, t)$ are introduced for the transformations above to be satisfied. Again, the factors $e / \hbar$ are present for convenience and the imaginary $i$ is a choice which enables $\phi$ and $\vec{A}$ to be real fields (we require $i \partial_{t}$ and $-i \vec{\nabla}$ to be Hermitian operators). The gauge transformation conditions imply that these fields obey the laws

$$
\begin{aligned}
\vec{A}(\vec{r}, t) & \rightarrow G \vec{A}(\vec{r}, t) G^{-1}+\frac{\hbar}{i e}(\vec{\nabla} G) G^{-1}=\vec{A}(\vec{r}, t)+\vec{\nabla} \alpha(\vec{r}, t) \\
\phi(\vec{r}, t) & \rightarrow G \phi(\vec{r}, t) G^{-1}-\frac{\hbar}{i e}\left(\partial_{t} G\right) G^{-1}=\phi(\vec{r}, t)-\partial_{t} \alpha(\vec{r}, t),
\end{aligned}
$$

where one recognizes the gauge tranformations of the vector and scalar potentials of electromagnetism.

Summarizing the discussion to this point, we made a local phase change to the wave function, but this does not leave the Lagrangian density invariant unless we compensate this change i.e. the terms brought about by derivating $\alpha$ can be cancelled by corresponding changes in the gauge fields. The terminology of covariant derivative refers to the fact that $\mathscr{D}_{t} \varphi$ and $\mathscr{D} \varphi$ are not invariant under a gauge transformation, but do transform in the same manner as the field $\varphi$ itself. The derivative operators $\mathscr{D}_{t}$ and $\overrightarrow{\mathscr{D}}$ themselve transform like vectors under a gauge transformation, $\mathscr{D}_{t} \rightarrow G \mathscr{D}_{t} G^{-1}$ and $\overrightarrow{\mathscr{D}} \rightarrow G \overrightarrow{\mathscr{D}} G^{-1}$. In the presence of an external gauge field, one way to make the Lagrangian density gauge invariant is the minimal coupling prescription, i.e. replace the ordinary derivatives by the covariant ones in the free matter field Lagrangian density (2),

$$
\begin{aligned}
\mathscr{L}_{\text {mat. }} & \equiv \mathscr{F}\left(\varphi, \varphi^{*}, \overrightarrow{\mathscr{D}} \varphi,(\overrightarrow{\mathscr{D}} \varphi)^{*}, \mathscr{D}_{t} \varphi,\left(\mathscr{D}_{t} \varphi\right)^{*}\right) \\
& =\mathscr{L}_{0}\left(\varphi, \varphi^{*}, \ldots\right)+\mathscr{L}_{\text {int. }}\left(\varphi, \varphi^{*}, \ldots, \phi, \vec{A}\right),
\end{aligned}
$$

where now there appears a supplementary interaction term, depending on both the "matter field" $\varphi$ and the gauge fields $\phi, \vec{A}$. This term appears because any term allowed by the gauge symmetry should appear. In principle higher order terms can also appear but one takes the minimal lowest orders. The price to pay to extend the so-called U(1) gauge invariance to local phase transformations is that it automatically generates the interactions of the charged matter field with the electromagnetic field, which is a strong self consistent check for the theory! Yang summarized the gauge invariance approach to the study of fundamental interactions by the expression "Symmetry dictates interaction" [31]

In terms of the gauge fields, the physical electric and magnetic fields $\vec{E}$ and $\vec{B}$ are defined as the commutators of covariant derivatives, $E_{k}=\frac{\hbar}{i e}\left[\mathscr{D}_{t}, \mathscr{D}_{k}\right]$ and $B_{k}=-\frac{1}{2} \frac{\hbar}{i e} \epsilon_{i j k}\left[\mathscr{D}_{i}, \mathscr{D}_{j}\right]\left(\epsilon_{i j k}\right.$ is the totally antisymmetric tensor which takes value +1 when $i j k$ is 123 up to circular permutations, it is -1 when $i j k$ is 213 up to circular permutations, and it is 0 when two or three indices are identical). In 4-dimensional notation, one introduces the Faraday tensor $F_{\mu \nu}=\frac{\hbar}{i e}\left[\mathscr{D}_{\mu}, \mathscr{D}_{\nu}\right]$ (with $\mathscr{D}_{\mu}=\partial_{\mu}+\frac{i e}{\hbar} A_{\mu}$ and the usual metric signature, $A_{\mu}=(\phi / c,-\vec{A})$ ) which transforms under gauge transformation as a vector, i.e. $F_{\mu \nu} \rightarrow G F_{\mu \nu} G^{-1}$. The Faraday tensor (hence the electric and magnetic fields) is thus gauge invariant in the Abelian $\mathrm{U}(1)$ gauge theory. The contribution of the free gauge fields to the Lagrangian density then follows simply from the following requirements: we demand a i) Lorentz invariant, ii) quadratic in the gauge fields derivatives (i.e. a kinetic energy of a standard form) and iii) gauge invariant quantity. Prescription i) is fulfilled if all space-time indices are contracted while ii) is nicely obtained from the square of the Faraday tensor. The quantity $F_{\mu v} F^{\mu v}$, which transforms under gauge transformation as $F_{\mu v} F^{\mu v} \rightarrow G F_{\mu v} F^{\mu v} G^{-1}$ satisfies all three conditions, since $G$ and $G^{-1}$ simplify each other in the present Abelian theory for which all quantities commute. This free gauge field Lagrangian density is known as the Schwarzschild Lagrangian density [32-34]),

$$
\mathscr{L}_{U(1)}=-\frac{1}{4 \mu_{0}} F_{\mu \nu} F^{\mu v}=\frac{1}{2} \varepsilon_{0} \vec{E}^{2}-\frac{1}{2 \mu_{0}} \vec{B}^{2} .
$$

The formulation of the principle of gauge invariance was stated in Yang and Mills work [4], where they generalize "phase invariance" of ordinary Quantum Mechanics of charged fields. They always refer to the case of electromagnetic theory in order to illustrate their arguments: We define isotopic gauge as an arbitrary way of choosing the orientation of the isotopic spin axes at all space-time points, in analogy with the electromagnetic gauge which represents an arbitrary way of choosing the complex phase factor of a charged field at all space-time points. We then propose that all physical processes (not involving the electromagnetic field) be invariant under the isotopic gauge transformation $\psi \rightarrow \psi^{\prime}, \psi^{\prime}=S^{-1} \psi$, where $S$ represents a space-time dependent isotopic spin rotation.

To preserve invariance one notices that in electrodynamics it is necessary to counteract the variation of $\alpha$ [the $\alpha$ of Yang and Mills is the same as our -e $\alpha$ in Eq. (5)] with $x, y, z$ and $t$ by introducing the electromagnetic field $A_{\mu}$ which changes under 
a gauge transformation as

$$
A_{\mu}^{\prime} \rightarrow A_{\mu}+\frac{1}{e} \partial_{\mu} \alpha
$$

In an entirely similar manner one introduces a $B$ field in the case of the isotopic gauge transformation to counteract the dependence of $S$ on $x, y, z$ and $t$. (...) The field equations satisfied by the twelve independent components of the $B$ field, which we shall call the $\mathbf{b}$ field, and their interaction with any field having an isotopic spin are essentially fixed, in much the same way that the free electromagnetic field and its interactions with charged fields are essentially determined by the requirement of gauge invariance.

It was also clearly stated by Utiyama [5]: The form of the interactions between some well known fields can be determined by postulating invariance under a certain group of transformations. For example, let us consider the electromagnetic interaction of a charged field $Q(x), Q^{*}(x)$. The electromagnetic interaction appears in the Lagrangian through the expressions $\partial_{\mu} Q-$ $i e A_{\mu} Q, \partial^{\mu} Q^{*}+i e A^{\mu} Q^{*}(1)$. The gauge invariance of this system is easily verified in virtue of the combinations of $Q, Q^{*}$, and $A_{\mu}$ in (1), if this system is invariant under the phase transformation $Q \rightarrow e^{i \alpha} Q, Q^{*} \rightarrow Q^{*} e^{-i \alpha} \alpha=$ const (2) [because the combination $\partial_{\mu} Q-i e A_{\mu} Q$ also transforms as Q]. Reversing the argument, the combination (1) can be uniquely introduced by the following line of reasoning. In the first place, let us suppose that the Lagrangian $L\left(Q, Q_{\mu}\right)$ is invariant under the constant phase transformation (2). Let us replace this phase transformation with the wider one (gauge transformation) having the phase factor $\alpha(x)$ instead of the constant $\alpha$. In order to make the Lagrangian still invariant under this wider transformation it is necessary to introduce the electromagnetic field through the combination (1). This combination and the transformation character of $A_{\mu}$ under the gauge transformation can be uniquely determined from the gauge invariance postulate of the Lagrangian $L\left(Q, Q_{, \mu}, A_{\mu}\right)$.

\section{Conserved currents and equations of motion in the presence of gauge fields}

In the present context of an Abelian gauge theory, the free gauge field contribution $\mathscr{L}_{U(1)}$ does not play any role in the expression of the conserved currents. This is due to the fact that the bosons (photons here), which are responsible for the (electromagnetic) interaction, do not carry the corresponding (electric) charge. We shall see later that this is no longer true in the non Abelian case. Nevertheless, the conserved current gets an additional term due to the presence of the interaction of the matter field $\varphi$ with the gauge potentials [35]. Indeed, since any local phase transformation is now a symmetry of the Lagrangian $\mathscr{L}_{\text {mat. }}=\mathscr{L}_{0}+\mathscr{L}_{\text {int. }}$, this is also true for global phase transformations and equation (4) now becomes

$$
\delta\left(\mathscr{L}_{0}+\mathscr{L}_{\text {int. }}\right)=0=-\alpha\left(\partial_{t} \rho+\vec{\nabla} \cdot \vec{J}\right)
$$

where the charge density $\rho(\vec{r}, t)$ is unchanged, but the current density acquires an additional term often called diamagnetic current density, $\vec{J}(\vec{r}, t)=\frac{-i e \hbar}{2 m}\left(\varphi^{*}(\vec{r}, t) \overrightarrow{\mathscr{D}} \varphi(\vec{r}, t)-\varphi(\vec{r}, t)(\vec{D} \varphi(\vec{r}, t))^{*}\right)=\vec{j}(\vec{r}, t)-\left(e^{2} / m\right) \vec{A}(\vec{r}, t) \varphi^{*}(\vec{r}, t) \varphi(\vec{r}, t)$. In terms of this conserved current density, the interaction term is often written to lowest powers in the gauge fields as $\mathscr{L}_{\text {int. }}=$ $\vec{J} \cdot \vec{A}-\rho \phi+O\left(\vec{A}^{2}\right)$, where all fields depend in the general case on the variables $(\vec{r}, t)$.

The total Langrangian density becomes

$$
\begin{aligned}
\mathscr{L}_{\text {tot. }}= & \mathscr{L}_{\text {mat. }}+\mathscr{L}_{U(1)} \\
= & i \hbar \varphi^{*}\left(\partial_{t}+\frac{i e}{\hbar} \phi\right) \varphi-\frac{\hbar^{2}}{2 m}\left[\left(\vec{\nabla}-\frac{i e}{\hbar} \vec{A}\right) \varphi\right]^{*}\left[\left(\vec{\nabla}-\frac{i e}{\hbar} \vec{A}\right) \varphi\right]-V \varphi^{*} \varphi \\
& +\frac{1}{2} \varepsilon_{0}\left(-\vec{\nabla} \phi-\partial_{t} \vec{A}\right)^{2}-\frac{1}{2 \mu_{0}}(\vec{\nabla} \times \vec{A})^{2}
\end{aligned}
$$

The equations of motion follow from Euler-Lagrange equations (1). For the matter field equation of motion, we perform the variation w.r.t. $\varphi^{*}(\vec{r}, t)$,

$$
0=\frac{\delta}{\delta \varphi^{*}} \int \mathscr{L}_{\text {tot. }} d^{3} r d t=\frac{\partial \mathscr{L}_{\text {mat. }}}{\partial \varphi^{*}}-\partial_{j} \frac{\partial \mathscr{L}_{\text {mat. }}}{\partial\left(\partial_{j} \varphi^{*}\right)}-\partial_{t} \frac{\partial \mathscr{L}_{\text {mat. }}}{\partial\left(\partial_{t} \varphi^{*}\right)}
$$

leading to

$$
i \hbar \partial_{t} \varphi=\frac{1}{2 m}(-i \hbar \vec{\nabla}-e \vec{A})^{2} \varphi+e \phi \varphi+V \varphi
$$


Those for the gauge fields also obey Euler-Lagrange equations, but follow from variations w.r.t. the gauge fields,

$$
\begin{aligned}
& 0=\frac{\delta}{\delta A_{k}} \int \mathscr{L}_{\text {tot. }} d^{3} r d t=\frac{\partial \mathscr{L}_{\text {mat. }}}{\partial A_{k}}-\partial_{j} \frac{\partial \mathscr{L}_{U(1)}}{\partial\left(\partial_{j} A_{k}\right)}-\partial_{t} \frac{\partial \mathscr{L}_{U(1)}}{\partial\left(\partial_{t} A_{k}\right)}, \\
& 0=\frac{\delta}{\delta \phi} \int \mathscr{L}_{\text {tot. }} d^{3} r d t=\frac{\partial \mathscr{L}_{\text {mat. }}}{\partial \phi}-\partial_{j} \frac{\partial \mathscr{L}_{U(1)}}{\partial\left(\partial_{j} \phi\right)}
\end{aligned}
$$

and the very definition of the charge and current densities $\rho=-\frac{\partial \mathscr{L}_{\text {mat }}}{\partial \phi}, \vec{J}=\frac{\partial \mathscr{L}_{\text {mat }}}{\partial \vec{A}}$. The Maxwell equations follow

$$
\begin{aligned}
& \vec{\nabla} \times(\vec{\nabla} \times \vec{A})-\varepsilon_{0} \mu_{0} \partial_{t}\left(-\vec{\nabla} \phi-\partial_{t} \vec{A}\right)=\mu_{0} \vec{J}, \\
& \vec{\nabla} \cdot\left(-\vec{\nabla} \phi-\partial_{t} \vec{A}\right)=\frac{\rho}{\varepsilon_{0}},
\end{aligned}
$$

the two equations without sources being automatically satisfied with the definition of the physical fields $\vec{E}$ and $\vec{B}$ in terms of the gauge fields. In the presence of other types of interactions of the matter field, there can occur new contributions to the charge and current densities in Maxwell equations as we will see later.

The gauge field theory approach thus appears as a very powerful tool in order to define properly the notion of conserved currents, the interaction of a matter field with an external (gauge) field, the equations of motion of these gauge fields, as well as some other subtle notions such as topological invariants [36]. This last notion is very useful for example in the description of the Aharonov-Bohm effect in which a charged particle moves in a pure gauge field (i.e. a region of space where the physical field $\vec{B}$ vanishes but the potential vector does not). In such a case, the gauge invariant phase of the wave function $\frac{i e}{\hbar} \oint \vec{A} d \vec{r}$ is responsible for the shift, proportional to the magnetic flux enclosed by the path, of the interference pattern produced by any kind of two-slit experiment [37].

\section{YANG-MILLS THEORY}

Our program is now to follow the same lines of derivation as previously outlined, but in the case of a SU(2) symmetry. This is done in many excellent textbooks $[38-43]$, so we will just motivate the theory and fix the notations that we will need in the application we have in mind to condensed matter physics. The U(1) gauge transformation introduced in the previous section was motivated by the fact that non relativistic (spinless) quantum mechanics deals with wave functions which are complex scalar fields and that physics is independent of the choice of global phase of this field. A complex scalar field can also be seen as two real scalar fields (the real and imaginary parts), and the gauge transformation thus appears as a rotation in the complex plane [44]. We can imagine that a more elaborate theory would require the introduction of more sophisticated mathematical objects, e.g. spinors [45] (or a set of complex scalar fields). In the simplest case, we will assume that the state of the particle is no longer described by a simple complex scalar field, but by a Pauli spinor consisting in two complex scalar fields usually written in the form of a "vector" $\psi=\left(\begin{array}{l}\varphi_{1} \\ \varphi_{2}\end{array}\right)$ and we will call SU(2) (special unitary group of $2 \times 2$ matrices with determinant 1 ) the corresponding gauge theory. This name comes from the fact that the objects acting on a two component spinor are two by two matrices which can all be written in terms of four basic ones, the unit matrix and the Pauli matrices. Now, we obviously have a set of 4 real scalar fields, but only 3 are in fact independent because the wave function must be normalized as $\int d^{3} r \psi^{\dagger} \psi<+\infty$ where the adjoint spinor is the "row vector" $\psi^{\dagger}=\left(\varphi_{1}^{*} \varphi_{2}^{*}\right)$. In the "internal" space called isospin space (name given by Eugene Wigner) where these three components live, a gauge transformation may be seen now as a rotation in a 3-dimensional space. Since the action of SU(2) operations rotate in three dimensional space and such rotations do not commute (order of rotations changes results) as in two dimensions, one says that the theory is non Abelian. This contrasts with the Abelian U(1) theory where complex scalars commute under multiplication. Such a transformation, acting on the spinor, may be written as

$$
\psi(\vec{r}, t) \rightarrow \exp (\operatorname{ig} \boldsymbol{\alpha} / \hbar) \psi(\vec{r}, t) .
$$

The constant $g$ plays the role of a new charge and all operators acting on the spinors are defined as $2 \times 2$ matrices (as expected), denoted here with bold font to emphasize their matrix nature, e.g. $\alpha$ stands for the contraction over the 
repeated index $a \tau^{a} \alpha^{a}=\tau^{1} \alpha^{1}+\tau^{2} \alpha^{2}+\tau^{3} \alpha^{3}$ where the $\tau^{a}$ are the generators of SU(2) (i.e. half the Pauli matrices),

$$
\tau^{1}=\frac{1}{2}\left(\begin{array}{ll}
0 & 1 \\
1 & 0
\end{array}\right), \tau^{2}=\frac{1}{2}\left(\begin{array}{cc}
0 & -i \\
i & 0
\end{array}\right), \tau^{3}=\frac{1}{2}\left(\begin{array}{cc}
1 & 0 \\
0 & -1
\end{array}\right) .
$$

The $\alpha^{a}$ 's are the parameters of the transformation, they define the components of the rotation vector in the threedimensional isospin space.

The transformation (18) is a symmetry when the parameters $\alpha^{a}$ 's are just constants, hence a conservation equation follows from Noether theorem, since, as quoted by R. Mills, "The substance of the theorem, for our purposes, is that for every symmetry of nature there is a corresponding conservation law and for every conservation law there is a symmetry" [2]. and its extension to local gauge symmetry is the strategy originally proposed by Yang and Mills [46]: "The conservation of isotopic spin points to the existence of a fundamental invariance law similar to the conservation of electric charge. In the latter case, the electric charge serves as a source of electromagnetic field. An important concept in this case is gauge invariance which is closely connected with (1) the equation of motion of the electromagnetic field, (2) the existence of a current density, and (3) the possible interactions between a charged field and the electromagnetic field. We have tried to generalize this concept of gauge invariance to apply to isotopic spin conservation". Isotopic spin or (strong) isospin had been introduced earlier by Heisenberg in 1933 as a new conserved physical quantity in order to explain the close similarity of behaviour of protons and neutrons during strong interactions, e.g. the same cross section (apart from electromagnetic corrections) for interactions involving both types of particles, like $p+\pi^{-} \rightarrow n+\pi^{0}$ or $n+\pi^{+} \rightarrow p+\pi^{0}$. The nucleon was then considered as an isospin doublet.

The extension of the transformation (18) to local parameters $\alpha^{a}(\vec{r}, t)$, faces the problem that exactly like in the case of $\mathrm{U}(1)$ symmetry, the space and time derivatives of the spinor do not obey the same transformation law (18) as the spinor itself does. As we mentioned before the simple gradient applied to the spinor is not gauge invariant, and thus is not a valid building block for the Lagrangian. The alternative is to come up with a recipe for a new derivative, the covariant derivative, which after having acted on a spinor, behaves also like a spinor (as one should expect, the derivative of a spinor is another spinor).

A space covariant derivative has to be defined as

$$
\overrightarrow{\mathscr{D}}=\vec{\nabla} 1-\frac{i g}{\hbar} \overrightarrow{\mathbf{W}}
$$

or in component form $\mathscr{D}_{k}=\partial_{k} 1-\frac{i g}{\hbar} \tau^{a} \mathrm{~W}_{k}^{a}$, and a covariant time derivative is similarly introduced by,

$$
\mathscr{D}_{t}=\partial_{t} 1+\frac{i g}{\hbar} \boldsymbol{\chi}=\partial_{t} 1+\frac{i g}{\hbar} \tau^{a} \chi^{a} .
$$

1 is the $2 \times 2$ identity matrix. The non-Abelian gauge potentials $\mathrm{W}_{k}^{a}$ and $\chi^{a}$ carry an isospin index, and they obey specific transformation laws through local gauge transformations.

\begin{tabular}{ccc}
\hline$U(1)$ & Global gauge transformations & $S U(2)$ \\
\hline$i \hbar \varphi^{*} \dot{\varphi}-\frac{\hbar^{2}}{2 m}(\vec{\nabla} \varphi)^{*}(\vec{\nabla} \varphi)$ & free particle Lagrangian density $\mathscr{L}_{0}$ & $i \hbar \psi^{\dagger} \dot{\psi}-\frac{\hbar^{2}}{2 m}(\vec{\nabla} 1 \psi)^{\dagger}(\vec{\nabla} 1 \psi)$ \\
$i \hbar \partial_{t} \varphi=-\frac{\hbar^{2}}{2 m} \vec{\nabla}^{2} \varphi$ & free particle equation of motion & $i \hbar \partial_{t} 1 \psi=-\frac{\hbar^{2}}{2 m} \vec{\nabla}^{2} 1 \psi$ \\
$\varphi \rightarrow \exp (i e \alpha / \hbar) \varphi$ & gauge transf. & $\psi \rightarrow \exp \left(\frac{i}{\hbar} g \alpha\right) \psi$ \\
$\partial_{t} \rho+\vec{\nabla} \cdot \vec{j}=0$ & continuity equation & $\partial_{t} \rho^{a}+\vec{\nabla} \cdot \vec{j}^{a}=0$ \\
$\rho=e \varphi^{*} \varphi$ & charge density & $\rho^{a}=g \psi^{\dagger} \tau^{a} \psi$ \\
$\vec{j}=\frac{-i e \hbar}{2 m}\left(\varphi^{*} \vec{\nabla} \varphi-\varphi \vec{\nabla} \varphi^{*}\right)$ & conserved current & $\vec{j}^{a}=\frac{-i g \hbar}{2 m}\left(\psi^{\dagger} \tau^{a}(\vec{\nabla} \psi)-(\vec{\nabla} \psi)^{\dagger} \tau^{a} \psi\right)$ \\
\hline
\end{tabular}

Table 1: Comparison between U(1) and SU(2) global gauge invariance. 
Once the non-Abelian gauge potentials have been defined, we can form a field tensor and a gauge covariant Lagrangian density in order to allow for a simple derivation of the conserved current density following from Noether theorem, and essentially follow similar lines of derivation than in the previous section. In order not to repeat all calculations, we present the analogies between $\mathrm{U}(1)$ and $\mathrm{SU}(2)$ theories in two tables. The properties associated to global gauge invariance are listed in the first table, while the consequences of local gauge invariance follow in the next table.

Let us briefly comment upon the results listed in these tables. In Table 1 there is a very clear one to one correspondence between the quantities defined in the two gauge theories. When one invokes global gauge invariance in the $\mathrm{SU}(2)$ case, isospin is inert fixed in a certain direction and everything operates as if charge is the only physical quantity. This is consistent with the interpretation of the Schrödinger equation not as an equation of motion for spinless particles, but for "two-component Pauli particles" in a spin eigenstate [47]. There are nevertheless important differences between the Abelian and the non-Abelian theories. First, the fact that the gauge potentials carry internal indices (this is the origin of the non-Abelian character), implies that the same indices are carried by the conserved charges, as well as by all other fields such as $\overrightarrow{\mathrm{E}}^{a}$ and $\overrightarrow{\mathrm{B}}^{a}$, the non-Abelian analogues to the electric and magnetic fields defined in Table 2. Another essential difference is due to the fact that the gauge fields also carry the "charge" of the interaction. This has two main consequences (see Table 2): the conserved charge density and current density have, together with the matter part, a purely radiative (i.e. involving only the gauge fields) contribution and the equations of motion for the gauge fields are non-linear.

\begin{tabular}{|c|c|c|}
\hline$U(1)$ & $\begin{array}{l}\text { Local gauge } \\
\text { transformations }\end{array}$ & $S U(2)$ \\
\hline$\varphi \rightarrow \exp (i e \alpha(\vec{r}, t) / \hbar) \varphi=G \varphi$ & local gauge transf. & $\psi \rightarrow \exp \left(\frac{i}{\hbar} g \boldsymbol{\alpha}(\vec{r}, t)\right) \psi=\mathbf{G} \psi$ \\
\hline $\left.\begin{array}{rl}\vec{D} & =\vec{\nabla}-\frac{i e}{\hbar} \vec{A} \\
\mathscr{D}_{t} & =\partial_{t}+\frac{i e}{\hbar} \phi\end{array}\right\}$ & cov. derivatives & $\left\{\begin{array}{l}\overrightarrow{\mathscr{D}}=\vec{\nabla} 1-\frac{i g}{\hbar} \overrightarrow{\mathbf{W}} \\
\mathscr{D}_{t}=\partial_{t} 1+\frac{i g}{\hbar} \chi\end{array}\right.$ \\
\hline $\left.\begin{array}{l}\vec{A} \rightarrow \vec{A}+\vec{\nabla} \alpha \\
\phi \rightarrow \phi-\partial_{t} \alpha\end{array}\right\}$ & $\begin{array}{l}\text { gauge transf. } \\
\text { of potentials }\end{array}$ & $\left\{\begin{array}{l}\overrightarrow{\mathbf{W}} \rightarrow \overrightarrow{\mathbf{W}}+\vec{\nabla} \boldsymbol{\alpha}+\frac{i g}{\hbar}[\boldsymbol{\alpha}, \overrightarrow{\mathbf{W}}] \\
\chi \rightarrow \chi-\partial_{t} \boldsymbol{\alpha}+\frac{i g}{\hbar}[\boldsymbol{\alpha}, \chi]\end{array}\right.$ \\
\hline $\left.\begin{array}{l}E_{k}=\frac{\hbar}{i e}\left[\mathscr{D}_{t}, \mathscr{D}_{k}\right] \\
B_{k}=-\frac{\hbar}{2 i e} \epsilon_{i j k}\left[\mathscr{D}_{i}, \mathscr{D}_{j}\right]\end{array}\right\}$ & field strengths & $\left\{\begin{array}{l}\mathbf{E}_{k}=\frac{\hbar}{i g}\left[\mathscr{D}_{t}, \mathscr{D}_{k}\right] \\
\mathbf{B}_{k}=-\frac{\hbar}{2 i g} \epsilon_{i j k}\left[\mathscr{D}_{i}, \mathscr{D}_{j}\right]\end{array}\right.$ \\
\hline $\begin{array}{l}i \hbar \varphi^{*} \mathscr{D}_{t} \varphi-\frac{\hbar^{2}}{2 m}(\overrightarrow{\mathscr{D}} \varphi)^{*}(\overrightarrow{\mathscr{D}} \varphi) \\
\quad+\frac{\varepsilon_{0}}{2}\left(|\vec{E}|^{2}-c^{2}|\vec{B}|^{2}\right)\end{array}$ & Lagrangian $\mathscr{L}_{\text {tot. }}$ & $\begin{array}{l}i \hbar \psi^{\dagger} \mathscr{D}_{t} \psi-\frac{\hbar^{2}}{2 m}(\overrightarrow{\mathscr{D}} \psi)^{\dagger}(\overrightarrow{\mathscr{D}} \psi) \\
\quad+\frac{g}{2 c}\left(\overrightarrow{\mathrm{E}}^{a} \cdot \overrightarrow{\mathrm{E}}^{a}-c^{2} \overrightarrow{\mathrm{B}}^{a} \cdot \overrightarrow{\mathrm{B}}^{a}\right)\end{array}$ \\
\hline$\rho=e \varphi^{*} \varphi$ & cov. charge density & $\rho_{\text {mat. }}^{a}+\rho_{\text {rad. }}^{a}=g \psi^{\dagger} \tau^{a} \psi-\frac{g}{c} \epsilon_{a b c} \overrightarrow{\mathrm{W}}^{b} \cdot \overrightarrow{\mathrm{E}}^{c}$ \\
\hline $\begin{array}{c}\vec{J}=\frac{-i e \hbar}{2 m}\left(\varphi^{*}(\vec{\nabla} \varphi)-\varphi(\vec{\nabla} \varphi)^{*}\right) \\
-\frac{e^{2}}{m} \vec{A} \varphi^{*} \varphi\end{array}$ & $\begin{array}{l}\text { cov. conserved } \\
\text { current }\end{array}$ & $\begin{array}{l}\vec{J}^{a}+\overrightarrow{\mathcal{J}}^{a}=\frac{-i g \hbar}{2 m}\left(\psi^{\dagger} \tau^{a}(\vec{\nabla} \psi)-(\vec{\nabla} \psi)^{\dagger} \boldsymbol{\tau}^{a} \psi\right) \\
-\frac{g^{2}}{4 m} \overrightarrow{\mathrm{W}}^{a} \psi^{\dagger} \psi-\frac{g}{c} \epsilon_{a b c}\left(\chi^{b} \overrightarrow{\mathrm{E}}^{c}+\frac{1}{2} c^{2} \overrightarrow{\mathrm{W}}^{b} \times \overrightarrow{\mathrm{B}}^{c}\right)\end{array}$ \\
\hline$i \hbar \partial_{t} \varphi=\frac{1}{2 m}(-i \hbar \vec{\nabla}-e \vec{A})^{2} \varphi+e \phi \varphi$ & $\begin{array}{c}\text { matter field } \\
\text { equation of motion }\end{array}$ & $i \hbar \partial_{t} \mathbf{1} \psi=\frac{1}{2 m}(-i \hbar \vec{\nabla} \mathbf{1}-g \overrightarrow{\mathbf{W}})^{2} \psi+g \boldsymbol{\chi} \psi$ \\
\hline $\left.\begin{array}{l}\vec{\nabla} \times \vec{B}-c^{-2} \partial_{t} \vec{E}=\mu_{0} \vec{J} \\
\vec{\nabla} \cdot \vec{E}=\frac{\rho}{\varepsilon_{0}}\end{array}\right\}$ & $\begin{array}{l}\text { gauge fields } \\
\text { equations of motion }\end{array}$ & $\begin{array}{l}\vec{\nabla} \times \overrightarrow{\mathrm{B}}^{a}-c^{-2} \partial_{t} \overrightarrow{\mathrm{E}}^{a}=\frac{1}{g c}\left(\vec{J}^{a}+\overrightarrow{\mathcal{J}}^{a}\right) \\
\vec{\nabla} \cdot \overrightarrow{\mathrm{E}}^{a}=\frac{c}{g}\left(\rho_{\text {mat. }}^{a}+\rho_{\text {rad. }}^{a}\right)\end{array}$ \\
\hline
\end{tabular}

Table 2: Comparison between U(1) and SU(2) local gauge invariance.

Another subtle difference between the Abelian and the non Abelian case resides in which quantities are gauge invariant, which are gauge covariant, and which are simply gauge dependent. One is used to the case of the electromagnetic interaction for which the charge density and the total current density are gauge invariant (even though in the latter 
case the paramagnetic and diamagnetic contributions are separetaly not gauge invariant, their sum is). The electric and magnetic fields (or the Faraday tensor) are similarly gauge invariant and finally only the wave function is gauge covariant $\varphi^{\prime}=G \varphi$. The covariant derivative operator $\mathscr{D}_{\mu}$ introduced to satisfy the same covariance law $\left(\mathscr{D}_{\mu} \varphi\right)^{\prime} \equiv \mathscr{D}_{\mu}^{\prime} \varphi^{\prime}=G \mathscr{D}_{\mu} \varphi$ then tranforms as a vector $\mathscr{D}_{\mu}^{\prime}=G \mathscr{D}_{\mu} G^{-1}$ and this implies that the gauge potential, although a 4-vector, has a particular non-covariant gauge transformation $A_{\mu}^{\prime}=G A_{\mu} G^{-1}-\frac{\hbar}{i e}\left(\partial_{\mu} G\right) G^{-1}$. In the non Abelian situation, the same consideration applies for the spinor $\psi^{\prime}=\mathbf{G} \psi$ and the covariant derivative operator, $\mathscr{D}_{\mu}^{\prime}=\mathbf{G}_{\mu} \mathbf{G}^{-1}$, and this requires the gauge potential to obey a similar non-covariant gauge transformation $\mathbf{W}_{\mu}^{\prime}=\mathbf{G W}_{\mu} \mathbf{G}^{-1}-\frac{\hbar}{i g}\left(\partial_{\mu} \mathbf{G}\right) \mathbf{G}^{-1}$. But now the so-called curvature tensor $\mathbf{F}_{\mu v}$ transforms like a vector, $\mathbf{F}_{\mu \nu}^{\prime}=\mathrm{GF}_{\mu} \mathbf{G}^{-1}$, i.e. is subject to a rotation in the isospin space, hence the $\mathrm{SU}(2)$ magnetic and electric fields are not gauge invariant and are then unphysical in a gauge symmetric theory (for a discussion on the meaning of "physical meaning", see e.g. Ref. [48]). The non Abelian charge density and current density themselves are not gauge invariant, and under a gauge transformation, their matter and radiation content changes. Only the total non Abelian charge $Q^{a}=\int d^{3} r \rho^{a}(\vec{r}, t)$ is gauge invariant (and constant in time) provided that the volume of integration extends over large enough distances for the current to vanish.

\section{YANG-MILLS THEORY FOR SPIN-ORBIT INTERACTION}

\section{A. Preamble - the Zeeman interaction}

Electrons are particles which carry not only an electric charge, but also a spin $\frac{1}{2}$. In non-relativistic Quantum Mechanics, they obey the Pauli equation which is a generalization of the Schrödinger equation with an essential innovation: the existence of the spin, i.e. a new degree of freedom, coupled to space-time degrees of freedom in the Hamiltonian through interactions such as the spin-orbit interaction and the Zeeman interaction. In the presence of such interactions, spin is known not to be conserved, but spin carries angular momentum and the total angular momentum is conserved. This is at the origin e.g. of the Einstein - de Haas experiment that will be discussed later.

Although these statements are well understood physically, a clear formulation is appealing following the lines of derivation proposed in the quotation of Yang and Mills [46] in section[III] Here the starting point is given by the known interaction terms present in the Pauli equation, from which a consistent gauge theory is built. Such a formulation leads, as before, to a conservation law. Then, once the gauge transformations are extended to local changes, it leads to the equations of motion for the gauge fields.

In this introductory section, we will focus attention on the consequences of the Zeeman interaction between the magnetic moment associated to the spin of the electron $\frac{e}{m} \overrightarrow{\mathbf{s}}$ (with Landé factor $g_{e}=2$ and $\overrightarrow{\mathbf{s}}=\frac{1}{2} \hbar \vec{\sigma}$ ) and an external magnetic field

$\vec{B}$. The state of the particle is described by a Pauli spinor $\psi=\left(\begin{array}{c}\varphi_{\uparrow} \\ \varphi_{\downarrow}\end{array}\right)$, and the Hamiltonian reads as $\mathbf{H}=\frac{1}{2 m}(\vec{p}-e \vec{A})^{2} 1+$ $V 1-\frac{e}{m} \overrightarrow{\mathbf{s}} \cdot \vec{B}$. Following standard textbooks $[49,50]$ one can build a continuity equation where the charge density and the charge current density are defined according to

$$
\begin{aligned}
\rho(\vec{r}, t)= & e \psi^{\dagger}(\vec{r}, t) \psi(\vec{r}, t), \\
\vec{J}(\vec{r}, t)= & \frac{-i e \hbar}{2 m}\left(\psi^{\dagger}(\vec{r}, t)[\vec{\nabla} \psi(\vec{r}, t)]-\left[\vec{\nabla} \psi^{\dagger}(\vec{r}, t)\right] \psi(\vec{r}, t)\right) \\
& -\frac{e^{2}}{m} \vec{A} \psi^{\dagger} \psi+\frac{e}{m} \vec{\nabla} \times\left(\psi^{\dagger} \overrightarrow{\mathbf{s}} \psi\right) .
\end{aligned}
$$

As we had already anticipated, when a new interaction (here the Zeeman interaction) appears in the problem, the conserved current gets modified. This is a property that we have already seen at play when the minimal coupling to the external electromagnetic field was added to the free particle, where it led to the appearance of a diamagnetic contribution to the Noether conserved current density. Here, the rotor of the magnetization associated to the electron density produces an additional charge current density. Note that this last term may be forgotten during the standard derivation using the wave equation and its complex conjugate, since the divergence of a rotor vanishes, and some care must be taken to establish the full current density [51-53]. Nevertheless, this addititonal term, sometimes called "spin term" [52], is compulsory in order to obtain a conservation equation. It is thus also instructive to contemplate the spin density (e.g. in the direction of the external field $\vec{B}=B \vec{e}_{z}$ ), which may be naturally defined by $s^{z}(\vec{r}, t)=\psi^{\dagger} s^{z} \psi$. The $z$-component $s^{z}$ commutes with 
the Hamiltonian. This is a conserved quantity, and the corresponding continuity equation takes the form $\partial_{t} s^{z}+\vec{\nabla} \cdot \vec{J}^{z}=0$ with $\vec{J}^{z}=\frac{-i \hbar}{2 m}\left(\psi^{\dagger} \mathbf{s}^{z} \vec{\nabla} \psi-\left(\vec{\nabla} \psi^{\dagger}\right) \mathbf{s}^{z} \psi\right)$ the spin current density. This is a tensorial quantity, since it has a vector character (the current propagates in space) and depends also on the spin orientation. The conservation equation also follows from the use of Ehrenfest theorem, $\frac{d s^{z}}{d t}=(i \hbar)^{-1} \psi^{\dagger}\left[\mathbf{s}^{z}, \mathbf{H}\right] \psi+\psi^{\dagger} \partial_{t} \mathbf{s}^{z} \psi=0$. The other components of the spin however do not commute with the Hamiltonian and in this case the continuity equation gets additional terms. Altogether, one can write $\partial_{t} s^{a}+\vec{\nabla} \cdot\left(s^{a} \vec{v}\right)=\frac{e}{m}(\vec{s} \times \vec{B})_{a}$, where the RHS represents the torque exerted on the spin.

The spin is just a contribution to the total angular momentum and this latter quantity is of course conserved. Our discussion thus suggests that there should be a way to write a conservation equation where spin components would appear explicitly, together with other sources of angular momentum. The "spin density" components $s^{a}(\vec{r}, t)=\psi^{\dagger} \mathbf{s}^{a} \psi$ are, in a sense equivalent to the charge density, except that they carry the spin index $a$. Deriving such a conservation equation is the purpose of the next sections.

\section{B. Pauli equation and spin-orbit interaction}

We are now in a position to discuss completely the role of the spin-dependent relativistic corrections to the Schrödinger equation. The Pauli equation follows from the non relativistic limit of the Dirac equation [54],

$$
i \hbar \partial_{t} \Psi=\left[c \vec{\alpha} \cdot(\vec{p}-e \vec{A})+\beta m c^{2}+V\right] \Psi,
$$

where $\vec{\alpha}$ and $\beta$ are the $4 \times 4$ Dirac matrices and $\Psi$ is a 4 -component Dirac spinor. We let $\Psi=\left(\begin{array}{c}\psi \\ \xi\end{array}\right) e^{-i m c^{2} t / \hbar}$ to remove the rest energy of the electron. $\psi$ and $\xi$ both have two components. In the non relativistic limit, $\xi$ is a very small component $\xi \simeq \frac{1}{m c^{2}-V} c \vec{\sigma} \cdot(\vec{p}-e \vec{A}) \psi$, and $\psi$ satisfies

$$
i \hbar \partial_{t} 1 \psi=\mathbf{H} \psi \text {. }
$$

Here, $\vec{\sigma}$ is the vector of Pauli matrices $\vec{\sigma}=\sigma^{1} \vec{e}_{x}+\sigma^{2} \vec{e}_{y}+\sigma^{3} \vec{e}_{z}=\sigma^{a} \vec{e}_{a}$. Forgetting about the relativistic correction to the kinetic energy, the Pauli Hamiltonian is given by

$$
\begin{aligned}
\mathbf{H}=\underbrace{\frac{1}{2 m}(\vec{p}-e \vec{A})^{2} \mathbf{1}}_{\text {Non Relativistic KE }}+V \mathbf{1} \\
\quad-\underbrace{\frac{e \hbar}{2 m} \vec{\sigma} \cdot \vec{B}}_{\text {Zeeman term }}-\underbrace{\frac{e \hbar}{4 m^{2} c^{2}} \vec{\sigma} \cdot(\vec{E} \times(\vec{p}-e \vec{A}))-\frac{i e \hbar^{2}}{8 m^{2} c^{2}} \vec{\sigma} \cdot \vec{\nabla} \times \vec{E}}_{\text {So interaction }}+\underbrace{\frac{e \hbar^{2}}{8 m^{2} c^{2}} \vec{\nabla}^{2} \phi}_{\text {Darwin term }} .
\end{aligned}
$$

In the following, we will neglect the Darwin interaction which, like the relativistic correction to the kinetic energy, is spin-independent. The "substrate" potential $V$ will be denoted as $e \phi$, defining the scalar gauge potential. Let us note that the SO interaction is written in its more general form here, including the term proportional to the rotor of the electric field [54].

\section{Gauge theory of the spin-orbit interaction}

The fact that the SO interaction is linear in $\vec{p}$ enables one to gather it together with the Non Relativistic KE in a single squared term, $\frac{1}{2 m}[(\vec{p}-e \vec{A}) 1-g \overrightarrow{\mathbf{W}}]^{2}$ up to a correction quadratic in the spin operator. Since $\frac{1}{2} \sigma^{a}$ is the generator of $\operatorname{SU}(2)$ symmetry (denoted as $\tau^{a}$ in tables 1 and 2), we set $\vec{W}=\frac{1}{2} \sigma^{a} \vec{W}^{a}$, with $\vec{W}^{a}$ three ordinary vectors. Expanding the cross products in terms of antisymmetric tensors, the SO interaction in Eq. (26) may now be written as

$$
\mathbf{H}_{\mathrm{SO}}=-\frac{e \hbar}{4 m^{2} c^{2}}\left[\boldsymbol{\sigma}^{a} \epsilon_{a j k} E_{j} p_{k}+\frac{i \hbar}{2} \sigma^{a} \epsilon_{a j k} \partial_{j} E_{k}\right]
$$


and identified to the relevant contribution of the cross term in the Non Relativistic KE,

$$
\mathbf{H}_{\mathrm{SO}}=-\frac{g}{4 m} \sigma^{a}\left(p_{k} \mathrm{~W}_{k}^{a}+\mathrm{W}_{k}^{a} p_{k}\right)=\frac{i \hbar^{2}}{4 m} \sigma^{a}\left(2 \mathrm{~W}_{k}^{a} \partial_{k}+\left(\partial_{k} \mathrm{~W}_{k}^{a}\right)\right)
$$

provided that the new "SO" charge, here denoted as $g$, is identified to $g=\hbar$. It is thus natural to define a new gauge vector potential, depending on both space-time variables and spin degrees of freedom, $g \vec{W}=\frac{e \hbar}{4 m c^{2}} \vec{\sigma} \times \vec{E}[55]$ or in components

$$
\frac{1}{2} g \boldsymbol{\sigma}^{a} \overrightarrow{\mathbf{W}}^{a} \equiv \frac{1}{2} g \boldsymbol{\sigma}^{a} \mathbf{W}_{k}^{a} \vec{e}_{k}=\frac{e \hbar}{4 m c^{2}} \epsilon_{a j k} \boldsymbol{\sigma}^{a} E_{j} \vec{e}_{k} .
$$

The same identification is obtained if we include the electromagnetic vector potential. We see that the gauge vector components $\mathrm{W}_{k}^{a}$ carry two indices. The index $k$ here refers to a purely space index while the index $a$ is a spin index which also has a spatial meaning due to the fact that spin and space variables are linked in Quantum Mechanics (the index $a$ would be a purely internal isospin variable in the context of Yang-Mills theories of fundamental interactions). In order to avoid any confusion between the two indices, we will always denote as upperscript the spin indices (the position of the indices makes no difference, since we use a Euclidean metric for this non relativistic theory). Repeated indices are summed up, independently of their up or down position. The SO interaction is now essentially included in the KE through

$$
\begin{aligned}
& \text { Non Relativistic KE }+ \text { SO Interaction }= \\
& \frac{1}{2 m}\left(\vec{p} \mathbf{1}-e \vec{A} \mathbf{1}-\frac{e \hbar}{4 m c^{2}} \overrightarrow{\boldsymbol{\sigma}} \times \vec{E}\right)^{2}-\frac{e^{2} \hbar^{2}}{32 m^{3} c^{4}}|\overrightarrow{\boldsymbol{\sigma}} \times \vec{E}|^{2}
\end{aligned}
$$

Let us point out the important fact that the gauge vector potential has an inherent matrix structure, hence the underlying gauge theory is a non-Abelian one, called SU(2) due to the presence of the Pauli matrices, exactly like what was briefly presented in section [II]. The addititional term in equation (30) has the structure of a gauge symmetry breaking term [57] i.e. it changes when we perform a gauge transformation. This, we now know how to do for this theory, and its effect will appear in a later discussion.

In the case of the SO+Zeeman interactions of equation $(26)$, one has to build a full $\mathrm{U}(1) \times \mathrm{SU}(2)$ theory. This means that we contemplate the space of complex scalar and their products and the space of $2 \times 2$ matrices and their product. Both these spaces being independent. To take into account simultaneously the fact that the electric and magnetic fields are minimally coupled to the charge and to the spin degrees of freedom, we define the covariant derivatives

$$
\begin{aligned}
& \overrightarrow{\mathscr{D}}=\vec{\nabla} \mathbf{1}-\frac{i e}{\hbar} \vec{A} \mathbf{1}-i \overrightarrow{\mathbf{W}}, \\
& \mathscr{D}_{t}=\partial_{t} \mathbf{1}+\frac{i e}{\hbar} \phi \mathbf{1}+i \chi .
\end{aligned}
$$

where $\vec{A}$ is the electromagnetic vector potential and $\mathrm{W}_{k}^{a}=\frac{e}{2 m c^{2}} \epsilon_{a j k} E_{j}$, while $\phi$ is the usual electromagnetic scalar potential, and $\chi^{a}=-\frac{e}{m} B^{a}$. Note that we have used the simplification which occurs due to the fact that the non-Abelian charge is $g=\hbar$.

Under an infinitesimal local gauge transformation $G=\exp \left(i \frac{1}{2} \sigma^{a} \alpha^{a}\right) \simeq 1+i \frac{1}{2} \sigma^{a} \alpha^{a}$, the non Abelian gauge fields should transform according to

$$
\begin{aligned}
\chi \rightarrow \chi^{\prime} & =\mathbf{G}_{\chi} \mathbf{G}^{-1}-\frac{1}{i}\left(\partial_{t} \mathbf{G}\right) \mathbf{G}^{-1}=\frac{1}{2} \sigma^{a}\left(\chi^{a}-\epsilon_{a b c} \alpha^{b} \chi^{c}-\partial_{t} \alpha^{a}\right) \\
\mathbf{W} \rightarrow \mathbf{W}^{\prime} & =\mathbf{G W G}^{-1}+\frac{1}{i}(\vec{\nabla} \mathbf{G}) \mathbf{G}^{-1}=\frac{1}{2} \sigma^{a}\left(\vec{W}^{a}-\epsilon_{a b c} \alpha^{b} \overrightarrow{\mathbf{W}}^{c}+\vec{\nabla} \alpha^{a}\right) .
\end{aligned}
$$

The alert student should note that in this theory of the spin-orbit interaction there is no gauge freedom associated to $\mathrm{SU}(2)$ transformations (actually a small subset survives[10]) since the non-Abelian gauge fields are defined in terms of the physical Maxwell fields [55]. We will come back later to this point. Another peculiarity is due to the appearance in equation (30) of the so-called gauge symmetry breaking term (GSB) (the last term in the equation) that interferes with gauge symmetry. The full Lagrangian hence becomes $\mathscr{L}_{\text {tot. }}=\mathscr{L}_{0}+\mathscr{L}_{\text {int. }}+\mathscr{L}_{\mathrm{GSB}}+\mathscr{L}_{U(1)}+\mathscr{L}_{\mathrm{SU}(2)}$, where $\mathscr{L}_{\text {mat. }} \equiv$ $\mathscr{L}_{0}+\mathscr{L}_{\text {int. }}+\mathscr{L}_{\mathrm{GSB}}\left(\mathscr{L}_{\text {mat. }}\right.$ contains all terms involving the matter field $\left.\psi\right)$. We also have to add the free field contribution 
$\mathscr{L}_{S U(2)}$ (called Yang-Mills Lagrangian density), which only depends on the non-Abelian fields. This term is required by gauge symmetry and Lorentz invariance (for the theory to be automatically extended to a relativistic context).

$$
\begin{aligned}
\mathscr{L}_{\text {tot. }}=\underbrace{i \hbar \psi^{\dagger} \mathscr{D}_{t} \psi-\frac{\hbar^{2}}{2 m}\left(\mathscr{D}_{k} \psi\right)^{\dagger}\left(\mathscr{D}_{k} \psi\right)}_{\mathscr{L}_{0}+\mathscr{L}_{\text {int. }}}+\underbrace{\frac{\hbar^{2}}{2 m} \psi^{\dagger} \mathbf{W}_{k} \mathbf{W}_{k} \psi}_{\mathscr{L}_{\mathrm{SBB}}} \\
+\underbrace{\frac{\varepsilon_{0}}{2}\left(E_{k} E_{k}-c^{2} B_{k} B_{k}\right)}_{\mathscr{L}_{U(1)}}+\underbrace{\frac{\hbar}{2 c}\left(\mathrm{E}_{k}^{a} \mathrm{E}_{k}^{a}-c^{2} \mathrm{~B}_{k}^{a} \mathrm{~B}_{k}^{a}\right)}_{\mathscr{L}_{S U(2)}},
\end{aligned}
$$

with

$$
\begin{aligned}
& \overrightarrow{\mathrm{E}}^{a}=-\partial_{t} \overrightarrow{\mathrm{W}}^{a}-\vec{\nabla} \chi^{a}+\epsilon_{a b c} \chi^{b} \overrightarrow{\mathrm{W}}^{c}, \\
& \overrightarrow{\mathrm{B}}^{a}=\vec{\nabla} \times \overrightarrow{\mathrm{W}}^{a}+\frac{1}{2} \epsilon_{a b c} \overrightarrow{\mathrm{W}}^{b} \times \overrightarrow{\mathrm{W}}^{c},
\end{aligned}
$$

following from the definitions $\mathbf{E}_{k}=\frac{1}{i}\left[\partial_{t} \mathbf{1}+i \chi, \partial_{k} \mathbf{1}-i \mathbf{W}_{k}\right]$ and $\mathbf{B}_{k}=-\frac{1}{2 i} \epsilon_{i j k}\left[\partial_{i} \mathbf{1}-i \mathbf{W}_{i}, \partial_{j} \mathbf{1}-i \mathbf{W}_{j}\right]$ given in Table 2 . The dimensions of the fields are consistent with the coefficients appearing in the covariant derivatives, $\left[\chi^{a}\right]=\mathfrak{T}^{-1}$, $\left[\mathrm{W}_{k}^{a}\right]=\mathfrak{L}^{-1},\left[\mathrm{E}_{k}^{a}\right]=\mathfrak{L}^{-1} \mathfrak{T}^{-1}$ and $\left[\mathrm{B}_{k}^{a}\right]=\mathfrak{L}^{-2}$, where $\mathfrak{T}$ and $\mathfrak{L}$ are time and space dimensions. The fact that the pure nonAbelian field contribution $\mathscr{L}_{S U(2)}$ is gauge invariant is not a priori obvious due to the gauge co-variance of the physical fields in the non-Abelian situation $\mathrm{E}_{k} \rightarrow \mathrm{GE}_{k} \mathrm{G}^{-1}=\mathrm{E}_{k}+i \frac{1}{2} \alpha^{a}\left[\sigma^{a}, \mathrm{E}_{k}\right]$ and the similar transformation law for $\mathrm{B}_{k}$. Nevertheless, $\mathscr{L}_{S U(2)}$ being proportional to $\operatorname{Tr}_{\mu \nu} \mathrm{F}^{\mu \nu} \rightarrow \operatorname{Tr} \mathrm{FF}_{\mu \nu} \mathrm{F}^{\mu \nu} \mathbf{G}^{-1}$, it is obviously gauge invariant thanks to the invariance properties of the trace under cyclic reordering of the matrices. There remains the question of the physical origin of this term in the present situation. As argued by Tokatly [21], it has the correct form to account for the first SO corrections to the energy. In any case it is demanded by symmetry so it should be out there!

\section{Conserved currents}

Let us stress on the fact that, contrary to the results presented in Table 2 in the case of a SU(2) gauge symmetric theory (Lagrangian invariant under SU(2) gauge transformations), the so called dia-color contribution $-\frac{\hbar^{2}}{4 m} \overrightarrow{\mathrm{W}}^{a} \psi^{\dagger} \psi$ to the current density is exactly compensated in the present situation by the GSB contribution. The term dia-color was coined in this context by Tokatly and refers to the analogue of the diamagnetic current density, but for the non Abelian SO interaction [21]. The matter current $J_{k}^{a} \equiv \frac{\partial \mathscr{L}_{\text {mat }}}{\partial \mathrm{W}_{k}^{a}}$ finally simplifies from the expression in Table 2 to

$$
J_{k}^{a}=\frac{-i \hbar^{2}}{2 m}\left(\psi^{\dagger} \frac{1}{2} \sigma^{a}\left(\partial_{k} \psi\right)-\left(\partial_{k} \psi\right)^{\dagger} \frac{1}{2} \sigma^{a} \psi\right),
$$

while the radiation current $\mathscr{J}_{k}^{a} \equiv \frac{\partial \mathscr{L}_{S U(2)}}{\partial \mathrm{W}_{k}^{a}}$ is the same as indicated in the table,

$$
\mathscr{J}_{k}^{a}=-\frac{\hbar}{c} \epsilon_{a b c}\left(\chi^{b} \mathrm{E}_{k}^{c}+\frac{1}{2} c^{2} \epsilon_{i j k} \mathrm{~W}_{i}^{b} \mathrm{~B}_{j}^{c}\right) .
$$

The GSB term has no effect on the matter and radiation spin densities which remain those of the table,

$$
s_{\text {mat. }}^{a} \equiv-\frac{\partial \mathscr{L}_{\text {mat. }}}{\partial \chi^{a}}=\psi^{\dagger} \frac{1}{2} \hbar \sigma^{a} \psi
$$

and

$$
s_{\text {rad. }}^{a} \equiv-\frac{\partial \mathscr{L}_{S U(2)}}{\partial \chi^{a}}=-\frac{\hbar}{c} \epsilon_{a b c} \mathrm{~W}_{k}^{b} \mathrm{E}_{k}^{c} .
$$

The spin-dependent part in the interaction term $\mathscr{L}_{\text {int. }}$ is thus exactly $J_{k}^{a} \mathrm{~W}_{k}^{a}-s_{\text {mat. }}^{a} \chi^{a}$ (with no quadratic contribution this time). A very simple, but important consequence is that there is no spin current contribution proportional to the non Abelian gauge vector, i.e. no current transverse to the ordinary electric field, and the spin Hall conductivity vanishes automatically in the GSB scenario [10]. 


\section{E. Equations of motion}

In order to obtain the equations of motion for the gauge fields, we also have to take into account the last peculiarity we will consider of the present problem, i.e. the fact that the $\mathrm{U}(1)$ and the $\mathrm{SU}(2)$ gauge fields are not independent. There are two approaches we can follow to contemplate this dependence within the variational formulation: one either includes constraints using the Lagrange multipliers [55] method, which allows treating the different gauge fields as independent,

$$
\begin{aligned}
& S_{\text {constr. }}\left[\psi, \phi, A_{k}, \chi^{a}, \mathrm{~W}_{k}^{a}\right]= S\left[\psi, \phi, A_{k}, \chi^{a}, \mathrm{~W}_{k}^{a}\right] \\
&-\int d t d^{3} r {\left[\mu^{a}\left(\chi^{a}+\frac{e}{m} B^{a}\right)+\lambda_{i}^{a}\left(\mathrm{~W}_{i}^{a}-\frac{e}{2 m c^{2}} \epsilon_{a j i} E_{j}\right)\right], } \\
& S\left[\psi, \phi, A_{k}, \chi^{a}, \mathrm{~W}_{k}^{a}\right]=\int d t d^{3} r \mathscr{L}_{\text {tot. }}\left(\psi, \phi, A_{k}, \chi^{a}, \mathrm{~W}_{k}^{a}\right),
\end{aligned}
$$

or one has to include in the equations of motion an account of the second order derivatives in the U(1) gauge fields, e.g.

$$
\begin{aligned}
\frac{\delta S}{\delta \phi}=\frac{\partial \mathscr{L}_{\text {tot. }}}{\partial \phi}-\partial_{t} & \frac{\partial \mathscr{L}_{\text {tot. }}}{\partial\left(\partial_{t} \phi\right)}-\partial_{k} \frac{\partial \mathscr{L}_{\text {tot. }}}{\partial\left(\partial_{k} \phi\right)} \\
& +\partial_{t}^{2} \frac{\partial \mathscr{L}_{\text {tot. }}}{\partial\left(\partial_{t}^{2} \phi\right)}+2 \partial_{t} \partial_{k} \frac{\partial \mathscr{L}_{\text {tot. }}}{\partial\left(\partial_{t} \partial_{k} \phi\right)}+\partial_{i} \partial_{j} \frac{\partial \mathscr{L}_{\text {tot. }}}{\partial\left(\partial_{i} \partial_{j} \phi\right)}=0 .
\end{aligned}
$$

Let us illustrate these two approaches in the very simple case of electrostatics where the Lagrangian density reduces to $\mathscr{L}=\frac{1}{2} \varepsilon_{0}|\vec{E}|^{2}-\rho \phi$. Using the first route, the condition $\vec{E}=-\vec{\nabla} \phi$ is implemented in the action through the constraint $S=\int d^{3} r[\mathscr{L}-\vec{\lambda} \cdot(\vec{E}+\vec{\nabla} \phi)]$. Varying the action w.r.t. $\phi(\vec{E}$ being considered as independent of $\phi)$ leads to $\frac{\partial \mathscr{L}}{\partial \phi}+\vec{\nabla}$. $\frac{\partial}{\partial(\vec{\nabla} \phi)} \vec{\lambda} \cdot(\vec{E}+\vec{\nabla} \phi)=-\rho+\vec{\nabla} \cdot \vec{\lambda}=0$, while variation w.r.t. $\vec{E}$ produces the equation $\varepsilon_{0} \vec{E}-\lambda=\overrightarrow{0}$. The combination of the two equations recovers the usual Maxwell equation. The second approach is more straightforward in this simple case and leads to $\frac{\partial \mathscr{L}}{\partial \phi}-\vec{\nabla} \cdot \frac{\partial \mathscr{L}}{\partial(\vec{\nabla} \phi)}=-\rho-\varepsilon_{0} \vec{\nabla}^{2} \phi=0$, i.e. the Poisson equation.

The first route is by far simpler in the SO case. For the usual electric and magnetic fields, we obtain the following equations of motion (Maxwell equations):

$$
\begin{gathered}
\frac{\delta S_{\text {constr. }}}{\delta \phi}=\frac{\delta S_{\text {mat. }}}{\delta \phi}+\frac{\delta S_{U(1)}}{\delta \phi}-\frac{\delta}{\delta \phi} \int d t d^{3} r \lambda_{i}^{a}\left[\mathrm{~W}_{i}^{a}-\frac{e}{2 m c^{2}} \epsilon_{a j i} E_{j}\right] \\
=\frac{\partial \mathscr{L}_{\text {mat. }}}{\partial \phi}-\partial_{k} \frac{\partial \mathscr{L}_{U(1)}}{\partial\left(\partial_{k} \phi\right)}-\frac{e}{2 m c^{2}} \partial_{k} \frac{\partial}{\partial\left(\partial_{k} \phi\right)}\left(\lambda_{i}^{a} \epsilon_{a j i} E_{j}\right) \\
=-\rho_{\text {mat. }}+\varepsilon_{0} \partial_{k} E_{k}+\frac{e}{2 m c^{2}} \partial_{k} \epsilon_{a k i} \lambda_{i}^{a}, \\
=0 . \\
\begin{array}{c}
\frac{\delta S_{\text {constr. }}}{\delta A_{i}}=\frac{\delta S_{\text {mat. }}}{\delta A_{i}}+\frac{\delta S_{U(1)}}{\delta A_{i}}-\frac{\delta}{\delta A_{i}} \int d t d^{3} r\left[\mu^{a}\left(\chi^{a}+\frac{e}{m} B^{a}\right)+\lambda_{k}^{a}\left(\mathrm{~W}_{k}^{a}-\frac{e}{2 m c^{2}} \epsilon_{a j k} E_{j}\right)\right] \\
=\frac{\partial \mathscr{L}_{\text {mat. }}}{\partial A_{i}}-\partial_{k} \frac{\partial \mathscr{L}_{U(1)}}{\partial\left(\partial_{k} A_{i}\right)}-\partial_{t} \frac{\partial \mathscr{L}_{U(1)}}{\partial\left(\partial_{t} A_{i}\right)}+\frac{e}{m} \partial_{k} \frac{\partial}{\partial\left(\partial_{k} A_{i}\right)}\left(\mu^{a} B^{a}\right)-\frac{e}{2 m c^{2}} \partial_{t} \frac{\partial}{\partial\left(\partial_{t} A_{i}\right)}\left(\lambda_{k}^{a} \epsilon_{a j k} E_{j}\right), \\
=J_{i}-\varepsilon_{0} c^{2} \epsilon_{i j k} \partial_{j} B_{k}+\varepsilon_{0} \partial_{t} E_{i}-\frac{e}{m} \epsilon_{i k a} \partial_{k} \mu^{a}+\frac{e}{2 m c^{2}} \partial_{t} \epsilon_{i k a} \lambda_{k}^{a}, \\
=0 .
\end{array}
\end{gathered}
$$

In the first of these expressions, we can define a polarization in terms of the Lagrange multipliers,

$$
P_{k}=\frac{e}{2 m c^{2}} \epsilon_{a k i} \lambda_{i}^{a} .
$$

Note that the origin of the dielectric polarization has to be found in the fact that a moving magnetic moment $\vec{\mu}$ creates a dipolar moment $\sim \vec{v} \times \vec{\mu}[56]$. This dielectric polarization is associated to bound charges and there appears then a 
contribution $-\partial_{k} P_{k}$ to the total charge density. This polarization also contributes the second equation of motion through a term added to the ordinary current density, $\partial_{t} P_{i}$, as well as an additional term $\epsilon_{i k a} \partial_{k} m^{a}$, with

$$
m^{a}=-\frac{e}{m} \mu^{a},
$$

describing "Amperian" currents (regular charge currents).

The electric charge and current densities are modified by the SO+Zeeman terms and the usual Maxwell equations become:

$$
\begin{aligned}
& \vec{\nabla} \cdot \vec{E}=\frac{1}{\varepsilon_{0}}(\rho-\vec{\nabla} \cdot \vec{P}), \\
& \vec{\nabla} \times \vec{B}-\varepsilon_{0} \mu_{0} \partial_{t} \vec{E}=\mu_{0}\left[\vec{J}+\partial_{t} \vec{P}+\vec{\nabla} \times \vec{m}\right] .
\end{aligned}
$$

The variation with respect to the non-Abelian gauge fields leads to

$$
\begin{aligned}
\frac{\delta S_{\text {constr. }}}{\delta \chi^{a}} & =\frac{\delta S_{\text {mat. }}}{\delta \chi^{a}}+\frac{\delta S_{S U(2)}}{\delta \chi^{a}}-\frac{\delta}{\delta \chi^{a}} \int d t d^{3} r \mu^{b}\left(\chi^{b}+\frac{e}{m} B^{b}\right), \\
& =\frac{\partial \mathscr{L}_{\text {mat. }}}{\partial \chi^{a}}+\frac{\partial \mathscr{L}_{S U(2)}}{\partial \chi^{a}}-\partial_{k} \frac{\partial \mathscr{L}_{S U(2)}}{\partial\left(\partial_{k} \chi^{a}\right)}-\mu^{a}, \\
& =-s_{\text {mat. }}^{a}+\frac{\hbar}{2 c} \epsilon_{a b c} \mathrm{~W}_{k}^{b} \mathrm{E}_{k}^{c}+\frac{\hbar}{c} \partial_{k} \mathrm{E}_{k}^{a}-\mu^{a} \\
& =0 . \\
\frac{\delta S_{\text {constr. }}}{\delta \mathrm{W}_{i}^{a}}= & \frac{\delta S_{\text {mat. }}}{\delta \mathrm{W}_{i}^{a}}+\frac{\delta S_{S U(2)}}{\delta \mathrm{W}_{i}^{a}}-\frac{\delta}{\delta \mathrm{W}_{i}^{a}} \int d t d^{3} r \lambda_{k}^{b}\left(\mathrm{~W}_{k}^{b}-\frac{e}{2 m c^{2}} \epsilon_{b j k} E_{j}\right), \\
= & \frac{\partial \mathscr{L}_{\text {mat. }}}{\partial \mathrm{W}_{i}^{a}}+\frac{\partial \mathscr{L}_{S U(2)}}{\partial \mathrm{W}_{i}^{a}}-\partial_{k} \frac{\partial \mathscr{L}_{S U(2)}}{\partial\left(\partial_{k} \mathrm{~W}_{i}^{a}\right)}-\partial_{t} \frac{\partial \mathscr{L}_{S U(2)}}{\partial\left(\partial_{t} \mathrm{~W}_{i}^{a}\right)}-\lambda_{i}^{a}, \\
= & J_{i}^{a}-\frac{\hbar}{2 c} \epsilon_{a b c}\left[\chi^{b} \mathrm{E}_{i}^{c}+c^{2} \epsilon_{i j k} \mathrm{~W}_{j}^{b} \mathrm{~B}_{k}^{c}\right]-\hbar c \epsilon_{i j k} \partial_{j} \mathrm{~B}_{k}^{a}+\frac{\hbar}{c} \partial_{t} \mathrm{E}_{i}^{a}-\lambda_{i}^{a}, \\
= & 0 .
\end{aligned}
$$

We recover the radiation spin density $s_{\text {rad. }}^{a}=-\frac{\hbar}{c} \epsilon_{a b c} \mathrm{~W}_{k}^{b} \mathrm{E}_{k}^{c}$ and the radiation spin current density $\mathscr{J}_{i}^{a}=-\frac{\hbar}{c} \epsilon_{a b c}\left[\chi^{b} \mathrm{E}_{i}^{c}+\right.$ $\left.\frac{1}{2} c^{2} \epsilon_{i j k} \mathrm{~W}_{j}^{b} \mathrm{~B}_{k}^{c}\right]$ in terms of which the corresponding Yang-Mills-Maxwell equations become

$$
\begin{aligned}
& \vec{\nabla} \cdot \overrightarrow{\mathrm{E}}^{a}=\frac{c}{\hbar}\left(s_{\text {mat. }}^{a}+s_{\text {rad. }}^{a}+\mu^{a}\right), \\
& \vec{\nabla} \times \overrightarrow{\mathrm{B}}^{a}-\frac{1}{c^{2}} \partial_{t} \overrightarrow{\mathrm{E}}^{a}=\frac{1}{\hbar c}\left(\vec{J}^{a}+\overrightarrow{\mathcal{J}}^{a}-\vec{\lambda}^{a}\right) .
\end{aligned}
$$

The non Abelian equations without sources take the form (obtained directly from the definitions (35) and (36))

$$
\begin{aligned}
& \vec{\nabla} \times \overrightarrow{\mathrm{E}}^{a}+\partial_{t} \overrightarrow{\mathrm{B}}^{a}=\epsilon_{a b c}\left(\vec{\nabla} \times\left(\chi^{b} \overrightarrow{\mathrm{W}}^{c}\right)+\frac{1}{2} \partial_{t}\left(\overrightarrow{\mathrm{W}}^{b} \times \overrightarrow{\mathrm{W}}^{c}\right)\right), \\
& \vec{\nabla} \cdot \overrightarrow{\mathrm{B}}^{a}=\frac{1}{2} \epsilon_{a b c} \vec{\nabla} \cdot\left(\overrightarrow{\mathrm{W}}^{b} \times \overrightarrow{\mathrm{W}}^{c}\right) .
\end{aligned}
$$

From the Yang-Mills-Maxwell equations, one can form a continuity equation describing the conservation of the total angular momentum density. Taking the divergence of Eq. (52), and using Eq. (51), one gets

$$
\partial_{t}\left(s_{\text {mat. }}^{a}+s_{\text {rad. }}^{a}\right)+\vec{\nabla} \cdot\left(\vec{J}^{a}+\overrightarrow{\mathcal{J}}^{a}\right)=-\partial_{t} \mu^{a}+\vec{\nabla} \cdot \vec{\lambda}^{a} .
$$

The Lagrange multipliers formally appear in the continuity equation, but physically, the 1.h.s. contains all sources of angular momentum incorporated in the problem [55], i.e. from free electrons in the conduction band encoded in the spinor $\psi$ (the matter spin density), and from angular momentum transfered to the lattice via SO interaction (the radiation angular momentum density). So the conservation of angular momentum in the system reads as expected

$$
\partial_{t}\left(s_{\text {mat. }}^{a}+s_{\text {rad. }}^{a}\right)+\vec{\nabla} \cdot\left(\vec{J}^{a}+\overrightarrow{\mathcal{J}}^{a}\right)=0 .
$$

We note that in the case of a static and homogeneous problem, the Lagrange multipliers just take the simple form $\mu^{a}=$ $-\left(s_{\text {mat. }}^{a}+s_{\text {rad. }}^{a}\right)$ and $\vec{\lambda}^{a}=\vec{J}^{a}+\overrightarrow{\mathscr{J}}^{a}$. 


\section{EINSTEIN - DE HAAS EXPERIMENT REVISITED}

The Einstein-de Haas experiment is one of the famous experiments of the beginning of the XXth century based on gyromagnetic phenomena [58-60]. In this experiment, a cylinder made of a non magnetized ferromagnetic material is suspended to a torsion wire and an external magnetic field is applied along the cylinder's axis. The cylinder acquires a magnetization, because spins orient to the field lowering the energy of the system and at the same time, the cylinder starts a rotating motion. This effect became an efficient experimental method for the measurement of the gyromagnetic ratio of various materials and, for example, proved that magnetism of iron is essentially due to the spin degrees of freedom.

This experiment is well known, and well understood from very fundamental principles, since it relies on the conservation of angular momentum. When the material gets magnetized, the individual magnetic moments of the electrons point in a common direction, hence a total angular momentum appears which has to be compensated by an opposite angular momentum carried by the whole sample in order to conserve the initially vanishing value of the total angular momentum of the sample. Although this mechanism is very clear and unquestionable, there is no simple microscopic explanation of how this conservation of angular momentum is at play at the atomic level. See however[61] for a beautiful description of the dynamics of the effect in terms of elastic twist on the lattice induced by the rotation of localized spins (spin-rotation coupling) and the coupling to the anisotropy field. The latter anisotropy is in fact a consequence of the existence, at a deeper level, of spin-orbit interactions. The purpose of this section is to provide such a microscopic description. Our approach is based on the gauge field description of spin-orbit interactions. The interest of the gauge field theory here is to provide a natural way for the introduction of conserved currents carrying angular momentum.

An external magnetic field $\vec{B}=B \vec{e}_{z}$ is applied to the cylinder of ferromagnetic material initially non magnetized, suspended along its $z$-axis to a torsion wire (hence the appearence of a non-Abelian scalar field $\chi^{3}$ in the Maxwell-Yang-Mills approach). The material gets magnetized. The spins acquire a common orientation and the corresponding magnetization is called the matter spin polarization. The sample is subject to a torsion around the vertical axis, and the radiation counterpart is the angular momentum acquired by the lattice. We can understand the physical situation as the appearence of a matter spin polarization $s_{\text {mat. }}^{3}$ since the individual localized spins get polarized along the direction of the external magnetic field. As a consequence, $\vec{\nabla} \cdot \vec{E}^{3} \neq 0$ and the Yang-Mills electric field aquires a non vanishing component $\vec{E}^{3}$. The localized spins interact with the lattice ions through an SO interaction (responsible for example for crystalline anisotropy), hence some components of $\vec{W}^{a}$ do not vanish, creating, together with $\chi^{3}$ a radiation contribution to the spin polarization $s_{\text {rad. }}^{3} \sim \varepsilon_{3 b c} \overrightarrow{\mathrm{W}}^{b} \cdot \overrightarrow{\mathrm{E}}^{c}$.

Let us analyse the equations in order to see whether an equation of rotation of the lattice follows. Here, the lattice enters the problem via the SO interaction, i.e. via the electric field (the "true" internal electric field) which is "rigidly" associated to the lattice ions. Since $s_{\text {rad. }}^{3}=-(\hbar / c) \varepsilon_{3 b c} \mathrm{~W}_{k}^{b} \mathrm{E}_{k}^{c}$, and $\mathrm{E}_{k}^{c}=-\partial_{t} \mathrm{~W}_{k}^{c}+\epsilon_{c d e} \chi^{d} \mathrm{~W}_{k}^{e}$ (we assume here that $\chi^{d}=\chi^{3}$, determined by the applied magnetic field, is uniform) we obtain

$$
s_{\text {rad. }}^{3}=\frac{e^{2} \hbar}{4 m^{2} c^{5}}\left(\vec{E} \times \partial_{t} \vec{E}+\frac{e}{m}\left(|\vec{E}|^{2}+\left(E_{3}\right)^{2}\right) \vec{B}\right)_{3} .
$$

As we had noticed, the appearence of the radiation contribution to the polarization is connected to the SO interaction (hence the electric field). This equation self-consistently shows that the true electric field produced by the ions located at the lattice sites is subject to a motion of rotation around the axis $\vec{e}_{3}$ along which the external magnetic field was applied. This rotation of the electric field can only be caused by an equivalent rotation of the whole lattice, thus explaining the observed Einstein-de Haas effect. If we now take into account the magnetization acquired by the cylinder and the exchange interaction between the conducting electrons and the localized moments ( $J_{s d}$ coupling constant), $\vec{B}$ has to be changed into $\vec{B}_{\text {eff }}=\vec{B}+J_{s d} \mu_{0} \vec{M}$.

\section{CONCLUSION}

In this paper, we have shown that spin-orbit interaction is a suitable arena to introduce non-Abelian gauge field theory (namely here $\mathrm{SU}(2)$ gauge theory) and that many consequences, relevant in a condensed matter physics context can be drawn from the classical form of the theory. More specifically, we have discussed the conserved current associated to this theory, the equations of motion of the gauge field, and the role of the continuity equation in the discussion of the Einstein - de Haas experiment. The approach follows from a very general Yang-Mills-Maxwell theory which we believe is likely suitable for micromagnetic simulations of spin current and spin configurations in various materials such as conducting ferromagnets or 2DEGs. 


\section{Acknowledgments}

This work was supported by CNRS-Fonacit grant PI-2008000272, and by the support of Nancy-Université through an Invited Professor position (EM). EM also acknowledges support from the POLAR Foundation.

\section{References}

[1] A.D. Boozer, "Classical Yang-Mills theory," Am. J. Phys. 79, 925 (2011).

[2] R. Mills, "Gauge fields," Am. J. Phys. 57, 493 (1989).

[3] E.B. Sonin, "Spin currents and spin superfluidity," Adv. Phys. 59, 181 (2010).

[4] C.N. Yang and R.L. Mills, "Conservation of isotopic spin and isotopic gaguge invariance," Phys. Rev. 96, 191 (1954).

[5] R. Utiyama, "Invariant theoretical interpretation of interaction," Phys. Rev. 101, 1597 (1956).

[6] A.C. Dotson, "Yang-Mills groups and fields," Am. J. Phys. 34, 670 (1966).

[7] A. Rebei and O. Heinonen, "Spin currents in the Rashba model in the presence of nonuniform fields," Phys. Rev. B 73, 153306 (2006).

[8] P.Q. Jin, Y.Q. Li and F.C. Zhang, "SU(2) × U(1) unified theory for charge, orbit and spin currents," J. Phys. A: Math. Gen. 39, 7115 (2006).

[9] B.W.A. Leurs, Z. Nazario, D.I. Santiago and J. Zaanen, "Non-Abelian hydrodynamics and the flow of spin in spin-orbit coupled substances," Ann. Phys. 323, 907 (2008).

[10] E. Medina, A. López and B. Berche, "Gauge symmetry breaking and topological quantization for the Pauli Hamiltonian," Europhys. Lett. 83, 47005 (2008).

[11] B. Berche, N. Bolívar, A. López and E. Medina, "Gauge field theory approach to spin transport in a 2D electron gas," Cond. Matt. Phys. 12, 707 (2009).

[12] C.A. Dartora and G.G. Cabrera, "Generation of electric field by spin-currents in the U(1) $\times$ SU(2) gauge invariant Pauli-Schrödinger non-relativistic theory," Phys. Lett. A 374, 2596 (2010).

[13] T. Fujita, M.B.A. Jalil, S.G. Tan and S. Murakami, "Gauge fields in spintronics," J. Appl. Phys. 110, 121301 (2011).

[14] E.I. Rashba, "Properties of semiconductors with an extremum loop. 1. Cyclotron and combinational resonance in a magnetic field perpendicular to the plane of the loop," Sov. Phys. Solid State 2, 1109 (1960).

[15] G. Dresselhaus, "Spin-Orbit Coupling Effects in Zinc Blende Structures," Phys. Rev. 100, 580 (1955).

[16] R. Winkler, Spin-Orbit Coupling Effects in Two Dimensional Electron and Hole Systems (Springer), Berlin 2003.

[17] B. Berche, C. Chatelain and E. Medina, "Mesoscopic rings with Spin-Orbit interactions," Eur. J. Phys. 31, 1267 (2010).

[18] A.S. Goldhaber, "Comment on Topological quantum effects for neutral particles," Phys. Rev. Lett. 62, 482 (1989).

[19] V.P. Mineev and G.E. Volovik, "Electric dipole moment and spin supercurrent in superfluid ${ }^{3}$ He," J. Low Temp. Phys. 89, 823 (1992).

[20] J. Fröhlich and U.M. Studer, "Gauge invariance and current algebra in non-relativistic many-body theory," Rev. Mod. Phys. 65, 733 (1993).

[21] I.V. Tokatly, "Equilibrium Spin Currents: Non-Abelian Gauge Invariance and Color Diamagnetism in Condensed Matter," Phys. Rev. Lett. 101, 106601 (2008).

[22] W.K.H. Panofsky and M. Phillips, Classical electricity and magnetism (Dover), Mineola 1983.

[23] N.A. Doughty, Lagrangian interaction (Addison Wesley), Singapore 1990, chap. 9, 10.

[24] H.R. Brown and P. Holland, "Simple applications of Noether's first theorem in quantum mechanics and electromagnetism," Am. J. Phys. 72, 34 (2004).

[25] E. Noether, "Invariante Variationsprobleme," Nachr. kgl. Ges. Wiss. Göttingen Math. Physik Kl. pp235-257 (1918); English translation available at arXiv:physics/0503066, or in Y. Kosmann-Schwarzbach, The Noether theorems (Springer), New-York 2010.

[26] L. O'Raifeartaigh, The dawning of Gauge theory (Princeton University Press), Princeton 1997.

[27] L. O'Raifeartaigh and N. Straumann, "Gauge theory: Historical origins and some modern developments," Rev. Mod. Phys. 72, 1 (2000).

[28] A.C.T. Wu and C.N. Yang, "Evolution of the concept of vector potential in the description of fundamental interactions," Int. J. Mod. Phys. 21, 3235 (2006).

[29] A. Salam and J.C. Ward, "On a gauge theory of elementary interactions," Il Nuovo Cimento 19, 165 (1961).

[30] S.F. Novaes, Standard Model: An Introduction, hep-ph/0001283

[31] C.N. Yang, "Einstein's impact on theoretical physics," Phys. Today 33, 42 (1980).

[32] K. Schwarzschild, "Zur Elektrodynamik; I,” Nachr. ges. Wiss. Gottingen , 125 (1903).

[33] P.B. Pal and K.S. Sateesh, "The field strength and the Lagrangian of a gauge theory," Am. J. Phys. 58, 789 (1990). 
[34] H. Essén, "From least action in electrodynamics to magnetomechanical energy - a review," Eur. J. Phys. 30, 515 (2009).

[35] D.L. Karatas and K.L. Kowalski, "Noether's theorem for local gauge transformations," Am. J. Phys. 58, 123 (1990).

[36] T.T. Wu and C.N. Yang, "Concept of nonintegrable phase factors and global formulation of gauge fields," Phys. Rev. D 12, 3845 (1975).

[37] M. Peshkin and A. Tonomura, The Aharonov-Bohm Effect (Springer-Verlag), Berlin 1989.

[38] L.H. Ryder, Quantum Field Theory (Cambridge University Press), Cambridge 1985, chap. 3.

[39] S. Weinberg, The Quantum Theory of Fields, Vol. II (Cambridge University Press), Cambridge 1996, chap. 15.

[40] M.E. Peskin and D.V. Schroeder, Quantum Field Theory (Addison-Wesley), Reading 1995, chap. 15.

[41] C. Quigg, Gauge theories of the strong, weak, and electromagnetic interactions (Westview Press), Reading 1983, chap. 3, 4.

[42] I.J.R. Aitchison and A.J.G. Hey, Gauge theories in particle physics - vol II: QCD and the electroweak theory (Taylor and Francis), New-York 2004, chap. 12, 13.

[43] P. Ramond, Field theory: a modern primer (Westview Press), Reading 1990, chap. 6.

[44] K. Moriyasu, "Gauge invariance and hidden symmetries," Am. J. Phys. 49, 819 (1980).

[45] R. Penrose, "The mathematics of electron's spin," Eur. J. Phys. 18, 164 (1997).

[46] C.N. Yang and R.L. Mills, "Isotopic spin conservation and a generalized gauge invariance," Phys. Rev. 95, 631 (1954).

[47] D. Hestenes and R. Gurtler, "Consistency in the Formulation of the Dirac, Pauli and Schroedinger Theories," J. Math. Phys. 16, 573 (1975).

[48] G. Giuliani, "Vector potential, electromagnetic induction and 'physical meaning'," Eur. J. Phys. 31, 871 (2010).

[49] L.D. Landau and E.M. Lifshitz, Quantum mechanics (non relativistic theory), 3rd ed (Elsevier), Oxford $2010, \S 115$.

[50] A.S. Davydov, Quantum mechanics (Pergamon), Oxford 1965, §65.

[51] D. Hestenes, "Spin and uncertainty in the interpretation of quantum mechanics," Am. J. Phys. 47, 399 (1979).

[52] M. Nowakowski, "The quantum mechanical current of the Pauli equation," Am. J. Phys. 67, 916 (1999).

[53] M.S. Shikakhwa, S. Turgut and N.K. Pak, "Derivation of the magnetization current from the non-relativistic Pauli equation: A comment on "The quantum mechanical current of the Pauli equation" by Marek Nowakowski [Am. J. Phys. 67(10), 916 (1999)]," Am. J. Phys. 79, 1177 (2011).

[54] J.D. Bjorken and S.D. Drell, Relativistic quantum mechanics (McGraw Hill), New-York 1964, , chap. 4.

[55] B. Berche, E. Medina and A. López, "Spin superfluidity and spin-orbit gauge symmetry fixing," EPL 97, 67007 (2012).

[56] R.V. Krotkov, G.N. Pellegrini, N.C. Ford and A.R. Swift, "Relativity and the electric dipole moment of a moving, conducting, magnetized sphere," Am. J. Phys. 67, 493 (1999).

[57] K. Moriyasu, "Breaking of gauge symmetry: A geometrical view," Am. J. Phys. 48, 200 (1980).

[58] O.W. Richardson, "A mechanical effect accompanying magnetization," Phys. Rev. (Series I) 26, 248 (1908).

[59] S.J. Barnett, "Magnetization by rotation," Rev. Mod. Phys. 7, 129 (1935).

[60] V.Ya. Frenkel, "On the history of the Einstein-de Haas effect," Sov. Phys. Usp. 22, 580 (1979).

[61] R. Jaafar, E.M. Chudnovsky and D.A. Garanin, "Dynamics of the Einstein-de Haas effect: Application to a magnetic cantilever," Phys. Rev. B 79, 104410 (2009). 